\title{
Experimental Model of Gene Transfection in Healthy Canine Myocardium. Perspectives of Gene Therapy for Ischemic Heart Disease
}

\author{
Renato A. K. Kalil, Leonardo A. K. Teixeira, Eduardo T. Mastalir, Paulo Moreno, \\ Cecília H. Fricke, Nance B. Nardi \\ Porto Alegre, RS - Brazil
}

\begin{abstract}
Objective - To assess the transfection of the gene that encodes green fluorescent protein (GFP) through direct intramyocardial injection.
\end{abstract}

Methods - The pREGFP plasmid vector was used. The EGFP gene was inserted downstream from the constitutive promoter of the Rous sarcoma virus. Five male dogs were used (mean weight $13.5 \mathrm{~kg}$ ), in which $0.5 \mathrm{~mL}$ of saline solution ( $n=1)$ or $0.5 \mathrm{~mL}$ of plasmid solution containing 0.5 $\mu \mathrm{g}$ of $p R E G F P / \operatorname{dog}(n=4)$ were injected into the myocardium of the left ventricular lateral wall. The dogs were euthanized 1 week later, and cardiac biopsies were obtained.

Results - Fluorescence microscopy showed differences between the cells transfected and not transfected with pREGFP plasmid. Mild fluorescence was observed in the cardiac fibers that received saline solution; however, the myocardial cells transfected with pREGFP had overt EGFP expression.

Conclusion - Transfection with the EGFP gene in healthy canine myocardium was effective. The reproduction of this efficacy using vascular endothelial growth factor (VEGF) instead of EGFP aims at developing gene therapy for ischemic heart disease.

Keywords: gene therapy, gene transfection, ischemic heart disease

Instituto de Cardiologia do Rio Grande do Sul/Fundação Universitária de Cardiologia and Universidade Federal do Rio Grande do Sul

Mailing address: Renato A. K. Kalil - Unidade de Pesquisa do IC/FUC - Av. Princesa Isabel, 395 - 90620-001 - Porto Alegre, RS, Brazil - e-mail: pesquisa@ cardnet.tche.br English version by Stela Maris C. e Gandour
Ischemic heart disease accounts for considerable morbidity and mortality in our environment. In the last few years, advances have been incorporated into the therapeutic options for myocardial revascularization, among which is gene therapy with a remarkable potential to control ischemic heart disease. Despite the large number of gene therapy protocols involving different genes of therapeutic interest for the cardiovascular system, such as VEGF (vascular endothelial growth factor), VEC (VE-cadherin), FGF (fibroblast growth factor), and PLGF (placental growth factor) genes, some points still require further clarification regarding the technique of transfection of these genes to the nuclear material of the myocardial cell ${ }^{1}$.

Accurate assessment of the efficacy of the methods of transfection, monitoring, and regulation of transgene expression in a cell or target tissue still raises many doubts and uncertainties. The most commonly used strategy that aims at simplifying these problems uses plasmid vectors containing marker (reporter) genes capable of accurately reproducing the results of the methodology of gene transfer. Several marker genes have been used in protocols of gene transfer for different tissues, such as the cardiovascular system, or cell types both in vitro and in vivo ${ }^{2-4}$.

In 1994, Chalfie et al ${ }^{5}$ and Gubin et al ${ }^{6}$ described green fluorescent protein (GFP), isolated from Aequoria victoria, as an important marker for gene transfer and gene expression in different organisms. That protein, when activated by ultraviolet light (395-470 nm), emits an easily monitored green fluorescence $(509 \mathrm{~nm})$ not requiring cofactors, substrates, or additional markers. Therefore, GFP expression may be detected in a direct and extremely simple form with flow cytometry (FACS, fluorescence-activated cell sorting) and fluorescence microscopy ${ }^{5,7}$.

Some variants of this protein emitting a more intense fluorescence were developed to better detect the expression of the reporter gene. Of these variants, enhanced green fluorescent protein (EGFP) stands out due to its fluorescence 35 
times greater than that of the native protein ${ }^{8}$. The EGFP gene has been largely used as a marker in several organisms, tissues, and cell types ${ }^{3,9}$. This way, different protocols of gene transfer have been based on this gene, enabling new investigations and excellent perspectives for the treatment of different genetic and acquired diseases ${ }^{10-13}$. Therefore, we chose the gene that encodes EGFP for this experimental model.

The present study aims at assessing the feasibility of the transfer of the gene that encodes EGFP through direct intramyocardial injection and its detection in healthy canine myocardium.

\section{Methods}

Construction and purification of plasmid DNA - We used pREGFP plasmid vector (fig. 1) ${ }^{14}$ created by subcloning the EGFP gene obtained from commercial pEGFP-N1 plasmid (Clontech Lab., Palo Alto, CA, USA) (fig. 2) between the 5' KpnI-NotI 3' cloning sites of pREP9 plasmid vector (Invitrogen Corp., San Diego, CA, USA) (fig. 3), provided by Carlos F. Menck (Institute of Biomedical Sciences USP, SP, Brazil). The EGFP gene was inserted downstream from the constitutive promoter of the Rous sarcoma virus (RSV). As a plasmid vector based on sequences of the Epstein-Barr virus (EBV), pREP9 plasmid has an EBV origin of replication (oriP), the gene that encodes the EBV nuclear antigen (EBNA-1), and the gene that confers resistance to neomycin $\left(\right.$ neo $\left.^{\mathrm{R}}\right)$. Plasmid DNA was amplified in XL1-Blue cells (Stratagene, La Jolla, CA, USA) through the standard method of transformation with calcium chloride ${ }^{15}$ and was purified with the Qiagen Plasmid Midi-Prep commercial kit (Qiagen, Valencia, CA, USA) (fig. 4).

Dogs used and surgical model - Five male dogs of unknown breeds with a mean weight of $13.5 \mathrm{~kg}$ were selected at

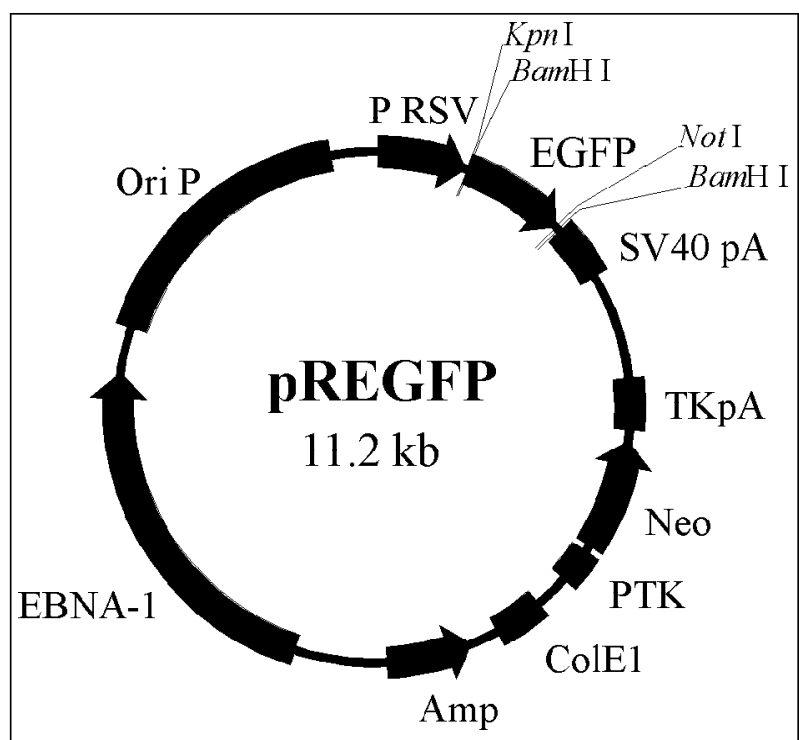

Fig. 1 - Sketch representing the pREGFP plasmid vector used during the injection procedure in the canine myocardium.

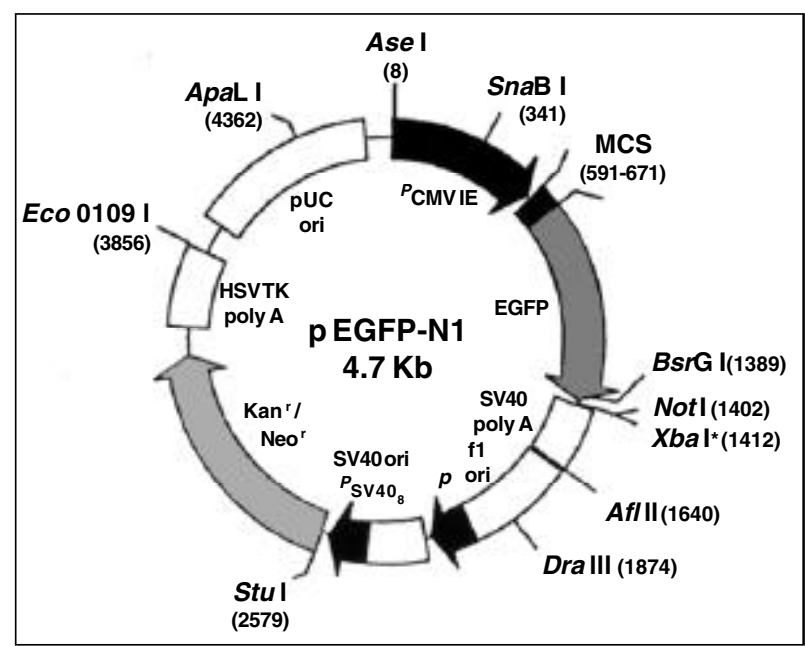

Fig. 2 - Sketch representing the pEGFP-N1 expression vector and its multiple cloning site (Clontech Lab).

the municipal kennel of the city of Porto Alegre to be used in this study. The dogs underwent vermifuge treatment with subcutaneous $1 \%$ ivermectin and received the octuple vaccine. The dogs were anesthetized with atropine $(0.044$ $\mathrm{mg} / \mathrm{kg}$ ), xylazine ( $2 \mathrm{mg} / \mathrm{kg})$, thiopental (10 mg/kg), $2 \%$ halothane (induction), and $1 \%$ halothane (maintenance). They also underwent antibiotic prophylaxis with ampicillin (40 mg/ $\mathrm{kg}) 20$ minutes prior to incision and fluid and electrolyte replenishment with lactated Ringer $(20 \mathrm{~mL} / \mathrm{kg} / \mathrm{h})$. They were intubated with orotracheal tubes of proper sizes and underwent assisted ventilation.

Preparation of the surgical field was performed with the dog restrained in the right lateral decubitus position, epilation of the left hemithorax, cutaneous antisepsis with iodine alcohol, and placement of sterile surgical fields. The heart was exposed through lateral thoracotomy in the $4^{\text {th }}$ left intercostal space and pericardiotomy. A mean volume of 0.5 mL of saline solution $(n=1)$ or plasmid solution containing $\operatorname{EGFP}(0.5 \mu \mathrm{g}$ of $\mathrm{pREGFP} / \mathrm{dog}-\mathrm{n}=4)$ was injected into the myocardium of the left ventricular lateral wall with an insulin syringe and needle. In the last 2 dogs, the plasmid solution was injected between 2 thread stitches left as a reference point to demarcate the exact injection site for directed sampling for the histological specimens. The pericardium, intercostal space, subcutaneous tissue, and skin were closed with separate stitches in their respective layers with monofilament thread 2-0.

After surgery, the dogs were taken to the kennel, where they were kept under supervision. One week after the procedure, the dogs were euthanized with magnesium sulfate (effect-depending dose), and their hearts were withdrawn through the previous thoracotomy. Specimens for biopsy were collected from the myocardial area previously demarcated between the 2 stitches left as reference points (last 2 dogs), and microscopic assessment of the myocardial cells was performed. In the first 2 dogs receiving the plasmid solution containing the EGFP reporter gene without demarcation of the injection site with stitches, the specimens 


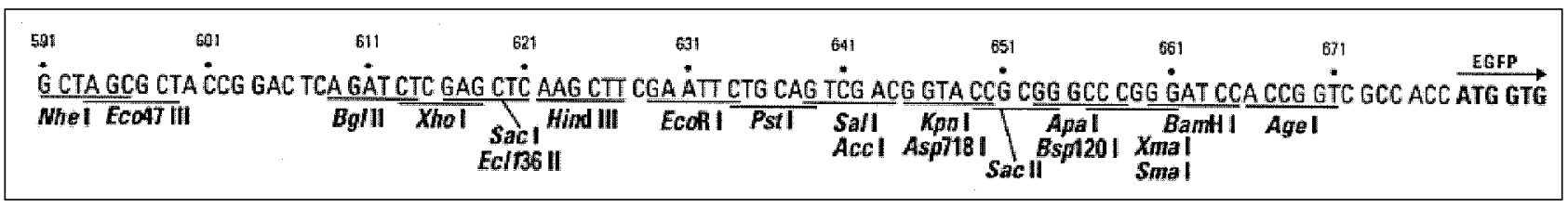

Fig. 3 - Sketch representing the pREP9 expression vector and its multiple cloning site (Invitrogen Corp).

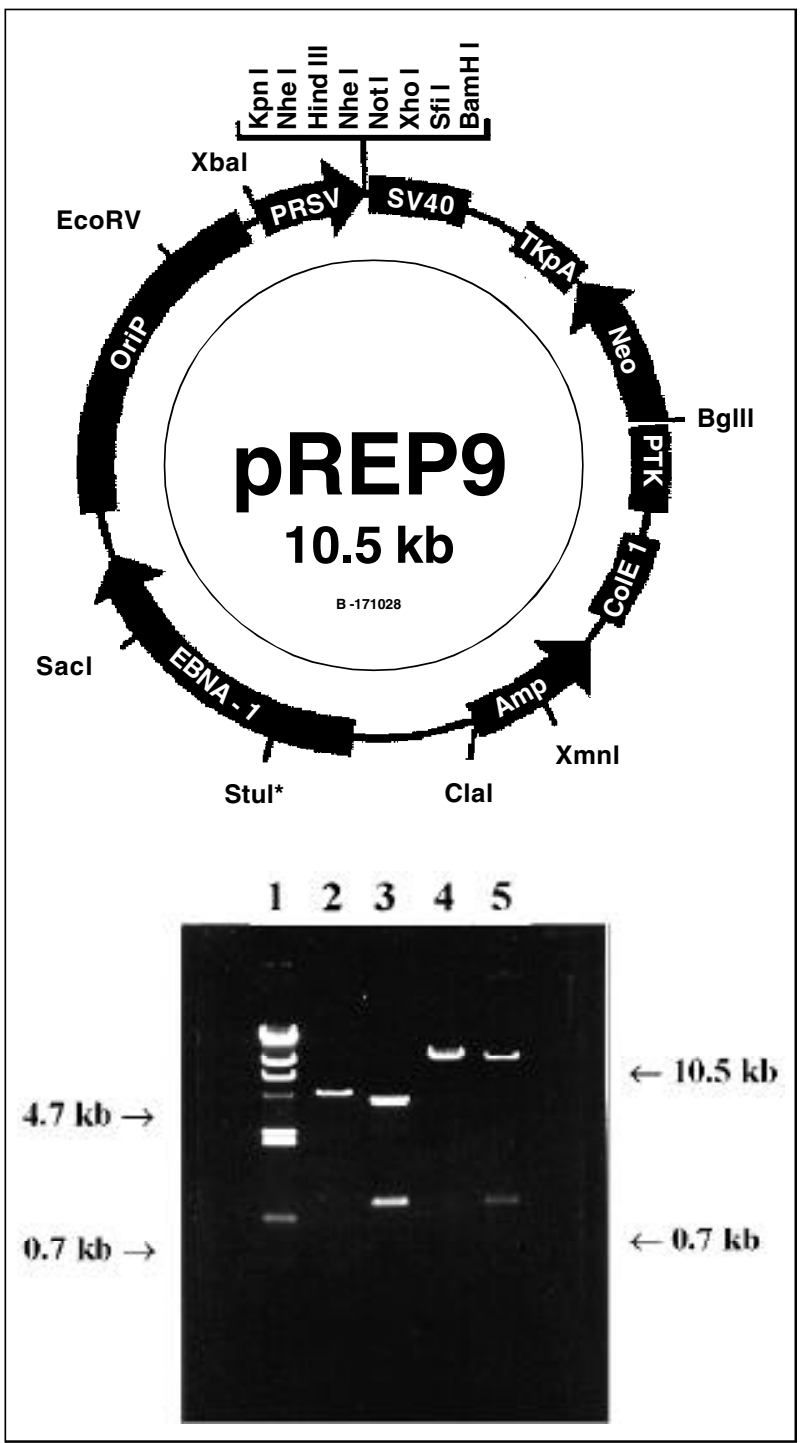

Fig. 4 - Photograph of the agarose gel for confirming subcloning of the EGFP gene in the pREP9 expression vector. $0.8 \%$ agarose gel stained with ethidium bromide. Line 1 - pattern of $\lambda$ molecular weight DNA/HindIII; line 2 - pEGFP-N1 expression vector linearized adjacent to the $K p$ nI enzyme; line 3 - pEGFP-N1 expression vector digested with the $K p n I$ and NotI enzymes; line 4 - pREP9 expression vector linearized adjacent to the $B a \mathrm{mHI}$ enzyme; line 5 - pREGFP recombinant clone digested with the $B a \mathrm{mHI}$ enzyme.

were collected at probable injection sites in the left ventricular lateral wall.

Detection and assessment of gene transfer - Fresh tissue fragments were preserved in antifreezing inclusion medium (Jung Tissue Freezing Medium, Leica Inst., Nussloch, Germany) and immediately cryopreserved in liquid nitrogen. After 24 hours, the samples were cut into $5.0 \mu \mathrm{m}$-thick sections with a LEICA CM 1800 cryostat (Leica Inst., Nussloch, Germany). The sections were mounted in glass slides for fluorescent microscopic assessment of the tissue transfected with the EGFP gene. To assess structural integrity, the myocardial tissue was stained according to the hematoxylineosin (HE) method. The microscopic analysis was performed with an Axiophot microscope (Carl Zeiss, Oberkochen, Germany)equipped with an argon laser with emission at $488 \mathrm{~nm}$ to activate the EGFP protein and a standard set of FITC filters (FT 510; BP 450/90; LP 520) to monitor fluorescent emissions. Microphotographs were obtained with an MC-3020N/M color videocamera(AVT-Horn, Aalen, Germany).

\section{Results}

During thoracotomy to remove the hearts, no visible alterations were observed on ectoscopy, on the epicardium, or on the myocardium at the site of injection of the plasmid solution. In regard to complications, $1 \mathrm{dog}$ had ventricular tachycardia during thoracotomy, prior to injection of the plasmid solution, but it was effectively reverted with lidocaine $(1.0 \mathrm{mg} / \mathrm{kg})$. The animals did not have fever, cardiac arrhythmias, pericardial effusion, or any other complications in the postoperative period, during which no cardiac enzyme was measured for control.

To assess the transgene expression in healthy canine myocardial cells, fluorescence microscopy was performed. Initially, the structure and integrity of the myocardial tissue were assessed in sections stained with HE, in which the myocardial tissue of the control animals and of those receiving injections with the genetic material maintained its structural integrity. However, sections of myocardial tissue analyzed under phase-contrast microscopy showed differences between the nontransfected cells (negative control) (fig. 5A) and those transfected with the pREGFP plasmid (positive control) (fig. 5B). The procedure of injecting gene material into the myocardial tissue of animals seems to have caused modifications in the conventional pattern of disposition of the myocardial fibers, resulting in some type of structural lesion (fig. 5B).

On fluorescence microscopy, an extremely discrete basal fluorescence was observed in the cardiac fibers that had not been transfected with the gene of interest (negative control) (fig. 5C). In contrast, in 1 (in which the histologic sections were performed exactly at the site delineated by the stitches left as a reference point in the myocardium) of the 4 animals transfected with pREGFP plasmid, myocardial cells with overt expression of the EGFP were observed on the $7^{\text {th }}$ day after DNA injection, when analyzed on fluores- 


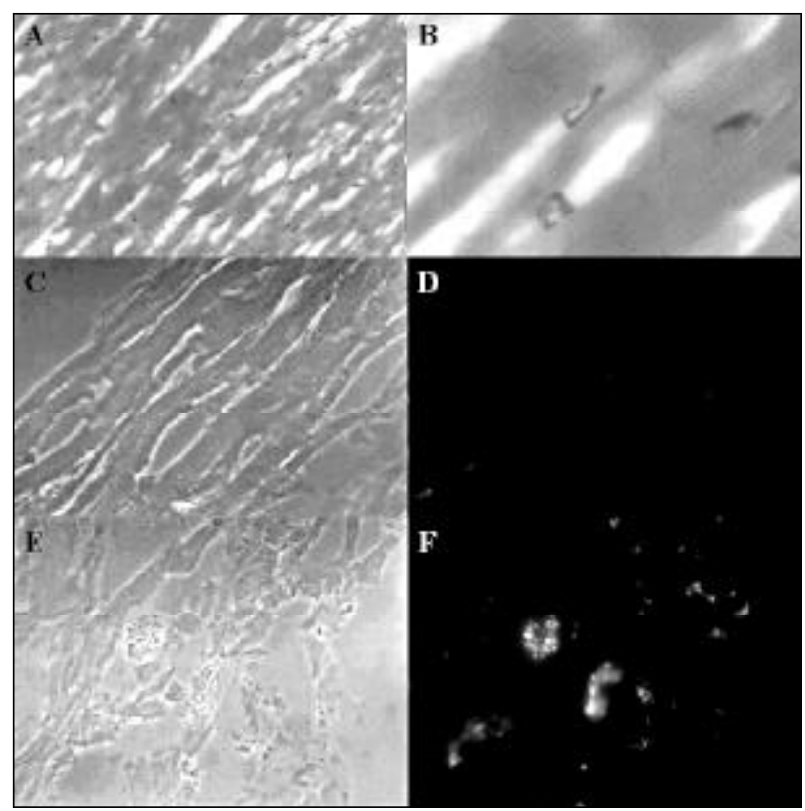

Fig. 5 - Photographs of phase-contrast microscopy (A and B) and fluorescence microscopy (C and D) of different cell sections of canine myocardium at a 1000x magnification. The left column depicts photos of sections of myocardial cells of control animals injected with saline solution (A and C). The right column depicts photos of sections of myocardial cells of animals injected with the solution of the gene material encoding EGFP. The myocardial cells transfected with the pREGFP plasmid expressing EGFP (D) were highlighted (arrow).

cence microscopy (fig. 5D). The distribution of EGFP inside the transfected cells was heterogeneous with some preferential sites of accumulation, mainly inside the nucleus. In addition, the expression of the transgene of interest was concentrated and extremely punctual inside the myocardial tissue, maybe coinciding with the site of plasmid injections. The intensity of fluorescence of the myocardial cells transfected with the pREGFP plasmid was also heterogeneous when visually compared with one another and with several processed sections. The percentage of transfected myocardial cells in the animal with overt EGFP expression was not assessed due to the methodological difficulty in isolating these cells, because of the high degree of cellular adhesion and tissue compactness.

\section{Discussion}

In the present experimental model, we report an efficient method of gene transfection for healthy canine myocardial cells, through direct injection of the pREGFP plasmid containing EGFP reporter gene. Several studies have shown that the EGFP protein does not have the capacity to trigger a significant immunological response in the host due to the absence of immunogenic epitopes ${ }^{11,12}$. Therefore, the structural alterations visualized on phase-contrast microscopy in the myocardial fibers of the animals treated with gene material seem to be a direct consequence of the invasive injection procedure. These alterations were not visualized on the material stained with $\mathrm{HE}$ or on the negative controls, probably due to matters of sampling.
The product of expression of the gene of interest was detected on fluorescence microscopy in the cells transfected with pREGFP plasmid, which showed considerable degrees of fluorescence as compared with the negative controls (cells receiving saline solution). The presence of EGFP protein occurred in a delineated region restricted to the site of puncture and injection of the gene material, showing the safety of the method and its possible reproducibility. The restriction of the detection of the transgene to the site of puncture may explain, at least partially, the detection of EGFP protein in only 1 of the 2 animals in which the injection was performed between the reference point stitches. This enabled the histologic sections to be performed more precisely and be restricted to the site of the procedure. In addition, the absence of a specific sign for cell externalization of the EGFP protein restricts its location to the interior of the effectively transfected cells ${ }^{3,5}$, and confirms its identification in delineated regions coinciding with the site of injection.

The EGFP protein in sections of normal myocardial cells in 1 of the 4 animals treated with the plasmid solution was detected 7 days after the gene transfection procedure, confirming that the expression of the gene of interest contained in pREGFP plasmid occurred up to that moment. Heterogeneity in the fluorescence intensity of the transfected myocardial cells is related to the efficiency of the individual transfection of each cell, ie, to the number of plasmid copies that each cell received at the moment of transfection ${ }^{16}$. Undoubtedly, whether transgene expression of myocardial cells can be monitored throughout time is a question that remains to be elucidated in further studies, so that its stability in the long run may be better evaluated ${ }^{6,11,12}$. Nevertheless, the results obtained in the 1-week period allow pertinent conclusions to be made and enable the continuity of this study through new approaches.

Therefore, the method proposed for EGFP gene transfection to healthy canine myocardium is functional and efficient. The development of this experimental model and the results obtained in this study provide tools for the use of genes of therapeutic interest. Our perspective points to the reproduction of this methodology in the experimental model reported for the use of the VEGF (vascular endothelial growth factor) therapeutic gene. Therefore, explanations about the stability of the transgene, capacity of VEGF extracellular signaling, and its consequent benefit for adjacent nontransfected cells, added to the possible adverse and physiological effects of this protein are certainly necessary. We aimed at the development of an experimental gene therapy protocol for the treatment of ischemic heart disease in our environment through the regional induction of angiogenesis through intramyocardial injections of specific plasmids that encode that growth factor.

\section{Acknowledgments}

The authors thank the following researchers for their fundamental participation in the development of this study: in Brazil - Gabriel Lorier Taranto, Maicon Becker, Beatriz 
Schaan, Carlos F. Menck, Antônio de Pádua da Silva Filho, Marcelo Meller Alievi, Carlos Marcos B. de Oliveira, Maria Isabel A. Edelweiss, Jorge Alberto Lopes; in the USA Thimothy Scott-Burden, Gerardo Gimenez-Sanchez; in
France - Manoel Vega; Márcio Scorsin. They also thank the Laboratório de Citogenética de Vertebrados of the Departament of Genetic of the Universidade Federal do Rio Grande do Sul (UFRGS) for its support.

\section{References}

1. Carmeliet P, Collen D. Molecular basis of angiogenesis-role of VEFG and VEcadherin. Ann N Y Acad Sci 2000; 902: 249-64.

2. Lamping KG, Rios CD, Chun JA, Ooboshi H, Davidson BL, Heistad DD Intrapericardial administration of adenovirus for gene transfer. Am J Physiol 1997; 272: H311-H17.

3. Plautz J, Day R, Dailey G, et al. Green fluorescent protein and its derivatives as versatile markers for gene expression in living Drosophila melanogaster, plant and mammalian cells. Gene 1996; 173: 83-7.

4. Svensson EC, Marshall DJ, Woodard K, et al. Efficient and stable transduction of cardiomyocytes after intramyocardial injection or intracoronary perfusion with recombinant adeno-associated virus vectors. Circulation 1999; 99: 201-5.

5. Chalfie M, Tu Y, Euskirchen G, Ward W, Prasher D. Green fluorescent protein as a marker for gene expression. Science 1994; 263: 802-5.

6. Gubin AN, Reddy B, Njoroge JM, Miller JL. Long-term, stable expression of green fluorescent protein in mammalian cells. Biochem Biophys Res Commun 1997; 236: 347-50.

7. Cheng L, Du C, Murray D, et al. A GFP reporter system to assess gene transfer and expression in human hematopoietic progenitor cells. Gene Ther 1997; 4: 1013-22.

8. Cormack B, Valdivia R, Falkow S. FACS-optimized mutants of green fluorescent protein (GFP). Gene 1996; 173: 33-8.

9. Zhang G, Gurtu V, Kain SR. An enhanced green fluorescent protein allows sensitive detection of gene transfer in mammalian cells. Biochem Biophys Res Commun 1996; 227: 707-11.
10. Bierhuizen M, Westerman Y, Visser T, Dimjati W, Wognum A, Wagemaker G. Enhanced green fluorescent protein as selectable marker of retroviral-mediated gene transfer in immature hematopoietic bone marrow cells. Blood 1997; 90: 3304-15.

11. Persons DA, Allay JA, Riberdy JM, et al. Use of the green fluorescent protein as a marker to identify and track genetically modified hematopoietic cells. Nat Med 1998; 4: 1201-5.

12. van Hennik PB, Verstegen MM, Bierhuizen MF, et al. Highly efficient transduction of the green fluorescent protein gene in human umbilical cord blood stem cells capable of cobblestone formation in long-term cultures and multilineage engraftment of immunodeficient mice. Blood 1998; 92 : 4013-22.

13. Kawada T, Shin WS, Nakatsuru Y, et al. Precise identification of gene products in hearts after in vivo gene transfection, using Sendai virus-coated proteoliposomes. Biochem Biophys Res Commun 1999; 259: 408-13.

14. Teixeira LAK, Fricke CH, Bonorino CB, Bogo MR, Nardi NB. An efficient gene transfer system for hematopoietic cell line using transient and stable vectors. $\mathrm{J}$ Biotechnol 2001 (no prelo).

15. Sambrook J, Fritsch E, Maniatis T. Molecular Cloning: a Laboratory Manual. 2nd edition. New York, NY: Cold Spring Harbor Laboratory Press; 1989.

16. Tseng WC, Haselton FR, Giorgio TD. Transfection by cationic liposomes using simultaneous single cell measurements of plasmid delivery and transgene expression. J Biol Chem 1997; 272: 25641-7. 